\title{
Force spectroscopy of a single artificial biomolecule bond: The Kramers' high-barrier limit holds close to the critical force
}

\author{
J. Husson, ${ }^{1, a)}$ M. Dogterom, ${ }^{1}$ and F. Pincet ${ }^{2, b)}$ \\ ${ }_{1}^{1}$ FOM Institute for Atomic and Molecular Physics (AMOLF), Kruislaan 407, 1098 SJ Amsterdam, \\ The Netherlands \\ ${ }^{2}$ Laboratoire de Physique Statistique de l'Ecole Normale Supérieure, Associé aux Universités \\ Paris 6 et Paris 7, UMR CNRS 8550, 24, rue Lhomond, 75231 Paris Cedex 05, France
}

(Received 14 October 2008; accepted 3 January 2009; published online 6 February 2009)

\begin{abstract}
We use a minimal system with a single micron-size bead trapped with optical tweezers to investigate the kinetics of escape under force. Surprisingly, the exponential decay of the off rate with the barrier energy is still valid close to the critical force. Hence, the high viscosity approximation derived by Kramers in the case of a high energy barrier holds even for an energy barrier close to the thermal energy. Several recent models describe a single biomolecule bond by a smooth single-barrier energy profile. When this approach is accurate enough, our result justifies the use of Kramers' approximation in the high-force regime, close to the critical force of the system, as done in recent single biomolecule bond studies. (C) 2009 American Institute of Physics. [DOI: 10.1063/1.3077010]
\end{abstract}

Since its development during the first half of the 20th century, ${ }^{1,2}$ Kramers' theory of the thermally activated escape from a potential well has been applied to a variety of physical systems. For example, in recent years, single biomolecule bonds have been a field in which Kramer's theory has emerged and become ubiquitous. Single molecule bonds provide cohesion to biological matter and are involved in a variety of fundamental biological processes. Their study has been made possible by the development of sensitive biophysical techniques [e.g., atomic force microscopy (AFM), optical tweezers (OTs), biomembrane force probe (BFP), laminar flow chamber]. Typical force spectroscopy experiments ${ }^{3}$ consist of exerting a force on a single biomolecule bond until it breaks. The goal is to extract from these measurements intrinsic parameters defining the bond. Ever since Bell's ${ }^{4}$ paper in 1978 and Evans ${ }^{5}$ connection of Bell's results with Kramers' theory, one dimensional (1D) energy landscapes $E(x)$ along a reaction coordinate $x$ have been used for the analysis of the experimental rupture of single molecular bonds. ${ }^{5}$ Clearly it is almost impossible to directly test the validity of this assumption, but the success and consistency of the results tend to prove this validity in more than just peculiar cases. In the simplest case, the energy landscape $E(x)$ presents a single energy barrier of height $E_{b}(0)$ relative to the energy minimum and located at a distance $x_{b}$ relative to the energy minimum. A common way to proceed is to apply a constant force $f$ to the bond and measure the lifetime of the bond. The lifetime distribution gives the off-rate $k_{\text {off }}(f)$ under this force $f$. The task for the experimentalist is then to extract from $k_{\text {off }}(f)$ information about $E(x)$. This analysis requires a model relating $k_{\text {off }}(f)$ to $E(x)$. Force spectroscopy models ${ }^{4,6-9}$ are usually based on Kramers' theory of the thermally activated escape from a potential well in the large viscosity limit. When the energy barrier is much larger than

\footnotetext{
${ }^{a)}$ Electronic mail: julien.husson@free.fr.

${ }^{b)}$ Electronic mail: pincet@lps.ens.fr.
}

the thermal energy $k_{B} T$, this theory gives the mean lifetime of the bond as

$$
\frac{1}{k_{\mathrm{off}}(f)}=\frac{1}{\nu_{D}(f)} \exp \left(\frac{E_{b}(f)}{k_{B} T}\right)
$$

where $E_{b}(f)$ is the energy barrier height under a given constant force $f$ and $\nu_{D}(f)$ the attempt frequency. The latter depends on the shape of $E(x)$, and also a priori on $f$,

$$
\nu_{D}=\frac{\sqrt{\kappa_{\min } \kappa_{\max }}}{2 \pi \gamma}
$$

where $\gamma$ is the friction coefficient and $\kappa_{\min }\left(\kappa_{\max }\right)$ is the curvature in the vicinity of the energy minimum (maximum) under the force $f$. Hence, Kramers' theory provides the basis for linking $k_{\text {off }}(f)$ to $f$, the tilted energy profile, and its local curvature. Theoretical work has addressed the behavior of single bonds close to the so-called critical force of the bond $f_{c}$, under which the energy barrier vanishes. However Eqs. (1) and (2) a priori are not valid when the energy barrier height approaches the thermal energy. ${ }^{7}$

In this paper we address experimentally the question of the range of validity of Eqs. (1) and (2). The nontrivial requirements of such a study are a fully determined $E(x)$ and a way to exert a controlled force on the system. Here we present an approach that fulfills these conditions. We study a micrometric particle, whose Brownian motion obeys the Langevin equation. We show that Kramers' theory, which is based on the Langevin equation and several mathematical assumptions, ${ }^{1}$ still provides an excellent approximation of the off-rate even when the energy barrier is close to $k_{B} T$.

We chose a minimal experimental system consisting of a single $1 \mu \mathrm{m}$ diameter silica bead trapped with OTs and submitted to a constant hydrodynamic drag force (Fig. 1). We call this system an artificial biomolecule bond by analogy to the situation where a single biomolecule bond is described by a smooth energy profile with a single energy barrier, and 


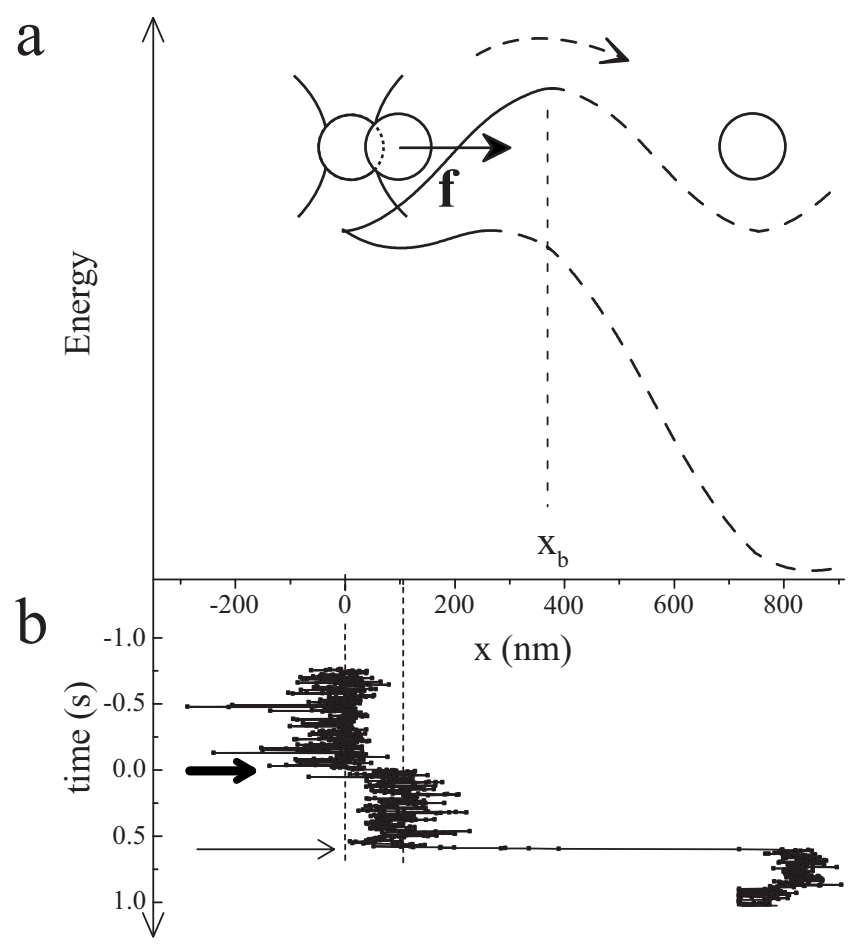

FIG. 1. (a) Schematic view of the setup and the energy profile. The trapped bead is represented without applied force, under force, and having escaped from the trapping potential. The energy profile is tilted when the force is applied (solid lines show the measured part of the energy profile; dashed lines show the part of the energy profile used to capture the bead after each escape). The dotted line indicates the energy barrier position $x_{b}$ under no force. (b) Time evolution of the $x$-coordinate of the bead during an escape. At $t=0.0 \mathrm{~s}$ the force is applied (short thick arrow). At $t \approx 0.6 \mathrm{~s}$ the bead escapes (long thin arrow). Dotted lines show the energy minimum position under no force and under applied force (here under force the energy minimum position undergoes $\mathrm{a} \approx 110 \mathrm{~nm}$ shift).

the escape of the bead over the energy barrier is the analog to the breaking of the bond. An infrared trapping laser (1064 $\mathrm{nm}, \mathrm{Nd}: \mathrm{YVO}_{4}$, Spectra-Physics, CA) was focused into the sample by a $100 \times / 1.3$ NA oil immersion objective. The laser beam was time shared with a $250 \mathrm{kHz}$ switching rate, using acousto-optical deflectors (AODs) (IntraAction DTD274HA6) in order to create a bistable potential. ${ }^{10,11}$ We studied the part of the potential for which $x \leq x_{b}$, consisting of the one-barrier energy profile. We used the part for which $x$ $\geq x_{b}$ to automatically capture the bead after each escape [Fig. 1(a)]. For position detection a low-power red laser $(633 \mathrm{~nm}$, HeNe, 1125P, Uniphase) was, after the AODs, superimposed on the infrared beam. The red laser light was focused on the $x$-axis in between the energy extrema positions $x=0$ and $x$ $=x_{b}$. The bead was imaged onto a quadrant photodiode allowing for measurement at a sampling frequency up to 30 $\mathrm{kHz}$. Wells with $7-\mu \mathrm{m}$-high walls were made on a acidcleaned glass coverslip with photoresist (SU-8, MicroChem Corp., Newton, MA) by using standard microlithography techniques. ${ }^{12}$ This way we could isolate a single bead in the $10 \mu \mathrm{l}$ distilled water sample kept at constant temperature $\left(25^{\circ} \mathrm{C}\right)$ by a water-circulating system. A hydrodynamic drag force was applied on the bead by translating the sample mounted on a piezoelectric stage. The translation speed was checked by analyzing differential interference contrast images of the wells at a sampling rate of $25 \mathrm{~Hz}$ with the use of

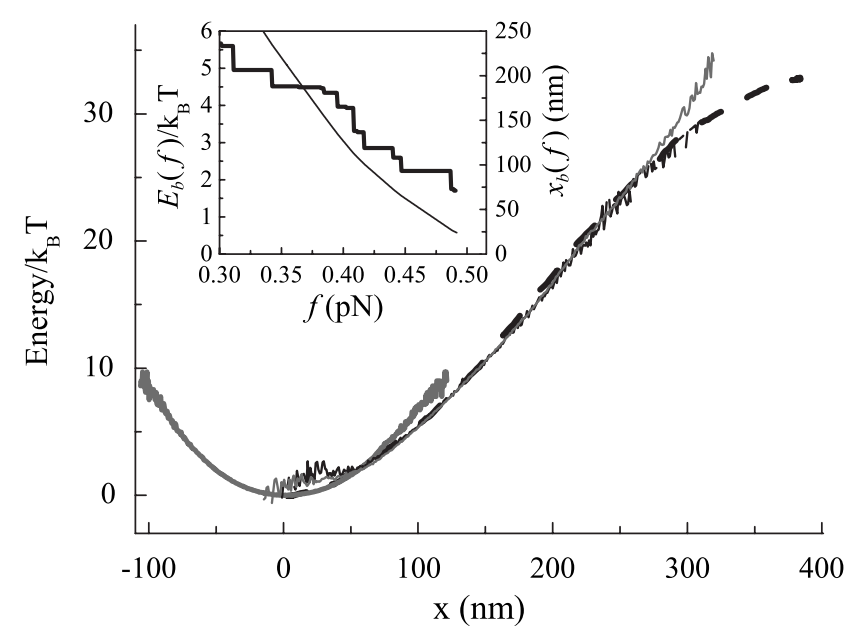

FIG. 2. Energy profile measured by two different methods. The first method consists of averaging $\dot{x}(t)$ over 100 trajectories (thick dashed black line), using the fact that $\langle\dot{x}\rangle$ is proportional to the energy gradient (Ref. 13): $\gamma\langle\dot{x}\rangle=\langle f\rangle=-d E / d x$. A second method for obtaining $E(x)$ consists of measuring the Boltzmann distribution of $x(t)$ at the energy minimum, under $f=0$ (thick solid gray line), $f=0.376 \mathrm{pN}$ (thin solid black line), and $f$ $=0.424 \mathrm{pN}$ (thin solid gray line). These latter measurements are consistent with the profile measured with the first method once the term $+f x$ with the corresponding $f$ is added to the measured potential under force. Inset: Forcedependent width $x_{b}(f)$ (thick line) and barrier height $E_{b}(f)$ (thin line) deduced from the measured $E(x)$ by adding a $-f x$ term.

a standard autocorrelation method (image processing software home written in IDL). Speeds were measured with a $0.1 \mu \mathrm{m} / \mathrm{s}$ error, which led to a $0.001 \mathrm{pN}$ error in force.

We used an energy profile (thick black dashed line in Fig. 2) with a $33 k_{B} T$ high energy barrier (barrier height was determined within an error equal to $\approx 3 k_{B} T$ under zero force and $\approx 1 k_{B} T$ upon approaching the critical force) and a width $x_{b} \approx 384 \mathrm{~nm}$. The ratio $E_{b} / x_{b}$ (Refs. 7 and 9) gives the order of magnitude of the critical force of the bond $f_{c}$. For this artificial bond, $f_{c}$ is a few tenths of piconewtons only, and a hydrodynamic drag force of this magnitude is easily achievable with a translation speed of the stage of few tens of $\mu \mathrm{m} / \mathrm{s}$. Hence, the study of the artificial bond leads us naturally to the high-force range.

In order to fully test Kramers' theory, we need to accurately describe the energy profile $E(x)$. As a first step, $x_{b}$ was determined by repeatingly releasing the bead from a given $x$-initial position. The initial position corresponded to the top of the energy barrier $x=x_{b}$ when, after release of the bead, the probability to be captured was the same for both energy minima of the bistable potential. Then the energy profile for $0 \leq x \leq x_{b}$ was first measured by using a method consisting of averaging several (100) trajectories $x(t)$ obtained by releasing the bead at the top of the energy barrier. Following the assumption that $x(t)$ obeys the Langevin equation, the averaged velocity $\langle\dot{x}\rangle$ is proportional to the energy gradient: ${ }^{13}$ $\gamma\langle\dot{x}\rangle=\langle f\rangle=-d E / d x$. An integration leads then to $E(x)$ (thick dashed black line in Fig. 2). The potential was also probed locally by measuring the Boltzmann distribution $p(x)$ $\propto \exp \left(-(E(x)-f x) / k_{B} T\right)$ of the $x$-coordinate of the bead ${ }^{11}$ under multiple applied force. By adding $+f x$ to the measured energy profile under the force $f$, we obtained within an approximately $20 \mathrm{~nm}$ origin shift (that we attribute to drift in 


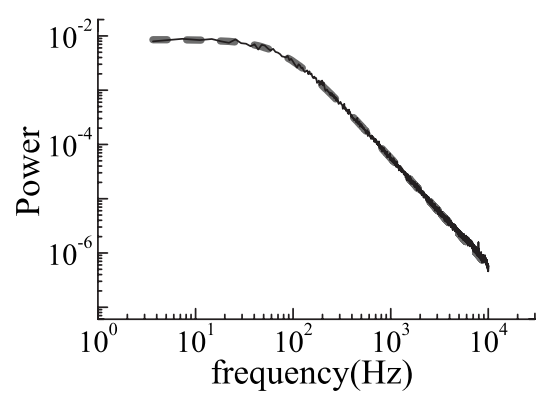

FIG. 3. Power spectrum of the fluctuations of $x$-coordinate of the bead at the energy minimum (solid black line) superimposed onto the fit with a Lorentzian form (thick dashed gray line) from which the corner frequency $\nu_{c}$ is extracted.

the setup) a reconstruction of the energy profile consistent with the result of the first reconstruction method (Fig. 2).

From $E(x)$, we were able to calculate the forcedependent width $x_{b}(f)$ and height $E_{b}(f)$ of the energy barrier (inset in Fig. 2). The curvature of the tilted energy profile $E(x)-f x$ in the vicinity of its minimum and maximum was roughly independent of the applied force $f$, hence a forceindependent attempt frequency. We used a more accurate measurement of $\kappa_{\min }$ by using a typical OT calibration method: ${ }^{14}$ the power spectrum of the fluctuations of the $x$-coordinate of the bead in the trapping potential (time sharing being still on) is a Lorentzian (Fig. 3), with a corner (roll-off) frequency $\nu_{c}=\kappa_{\min } / 2 \pi \gamma=81.2 \pm 0.2 \mathrm{~s}^{-1}$. This led to $\kappa_{\min }=4.81 \times 10^{-5} \pm 0.01 \times 10^{-5} \mathrm{pN} / \mathrm{nm}$. $\kappa_{\max }$ was obtained (making the same assumption of the system obeying the Langevin equation as above) by fitting the averaged trajectory $\langle x\rangle(t)$ to an exponential decrease close to the energy barrier: $\langle x\rangle(t)=x_{b} \exp \left(-\kappa_{\max } / \gamma t\right)$. This led to $\kappa_{\max }=3.55$ $\times 10^{-5} \pm 0.02 \times 10^{-5} \mathrm{pN} / \mathrm{nm}$. As a result, we obtained $\nu_{D}$ $=69.7 \pm 0.4 \mathrm{~s}^{-1}$. Finally, at $f=f_{c}$, both $x_{b}(f)$ and $E_{b}(f)$ vanish. We obtained from the measured potential $f_{c}$ $=0.49 \pm 0.01 \mathrm{pN}$. The knowledge of $E_{b}(f)$ and $\nu_{D}$ allowed us to calculate the predicted $k_{\text {off }}(f)$ using Kramers' approximation given by Eqs. (1) and (2) (solid line in Fig. 4).
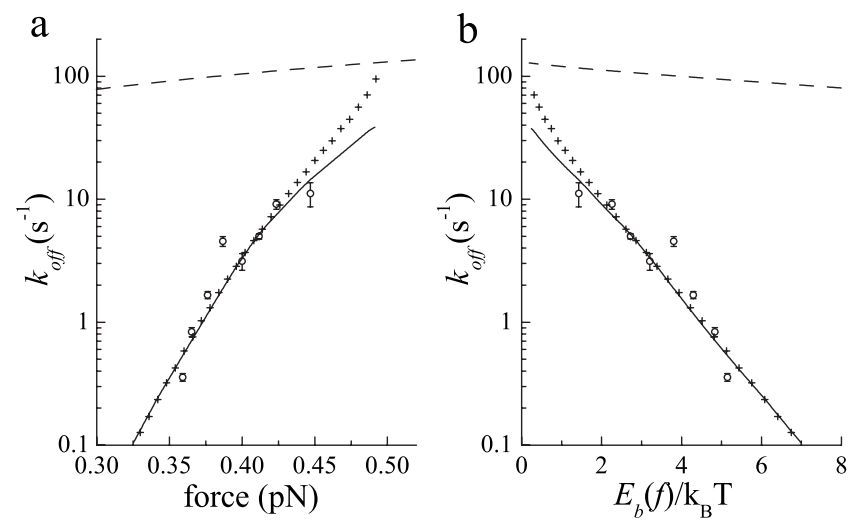

FIG. 4. Experimental (circles, bars are standard errors) and theoretical offrates. Solid line is Kramers' off-rate obtained from Eqs. (1) and (2), crosses represent the off-rate given by a MFPT approach with a $33.5 k_{B} T$ height of the energy barrier under zero force. Off-rates are plotted in (a) vs the applied force $f$ and (b) vs the barrier height $E_{b}(f)$ in units of $k_{B} T$. The dashed line represents the inverse of the travel time from $x=0$ to $x=x_{b}$ if the bead were to adopt the stage translation speed.
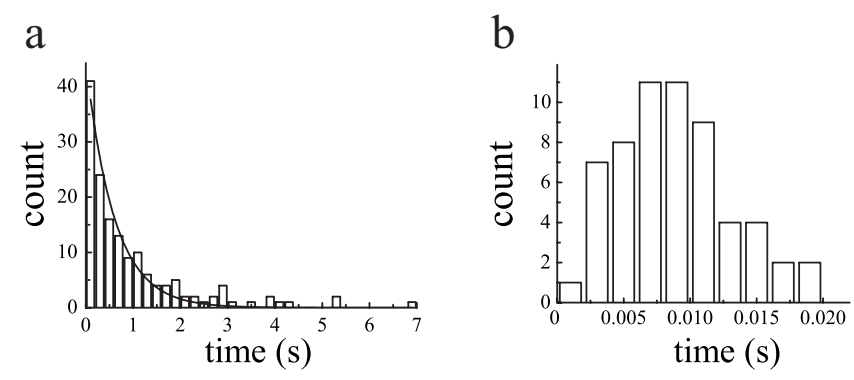

FIG. 5. Distribution of the escape time of the bead under different forces $f$. (a) $f=0.376 \mathrm{pN}$; (b) $f=0.589 \mathrm{pN}$, this force is beyond the critical force $f_{c} \approx 0.490 \mathrm{pN}$, and the escape time distribution ceases to be exponential.

Independently of the potential measurements, we measured the first escape time when the force was applied on the bead. For each applied force $f$, several escapes of the bead (typically 100) were recorded. The piezoelectric stage was translated at a constant speed over $40 \mu \mathrm{m}$ and then brought back to its initial position. If the bead had escaped during the translation, it was automatically captured by the second energy minimum composing the whole double-well potential. If the bead had not escaped during the translation, duration of successive attempts until bond breakage were added, following the assumption of a Markovian system. The escape time was defined as the first time of passage over the barrier, whose location was deduced from the measured potential. At the lowest energy barriers, the particle sometimes undergoes fluctuations slightly over the barrier without being captured by the above-mentioned second energy minimum. However, first and last passage times do not have different enough distributions to change our conclusions; in this paper we only show statistics of the first passage time. The experimental distributions of the escape time of the bead are exponential for all our measurements at $f<f_{c}$ [Fig. 5(a)], consistent with a first-order rate equation $\dot{P}(t)=-k_{\text {off }}(f) P(t)$, where $P(t)$ is the probability for the bond to stay intact after the force $f$ has been settled at time $t=0$. The transient regime caused by the onset of the stage translation (on the order of ten milliseconds) is always much shorter than the mean lifetime of the bond for $f<f_{c}$, so that its influence on the dynamics of the system is negligible, and the simple first-order rate equation under constant force holds from $t=0 \mathrm{on}$. For the measurement done at $f>f_{c}$, the time distribution ceases to be exponential [Fig. 5(b)], and the mean escape time approaches the travel time needed to get from $x=0$ to $x=x_{b}$ at the stage translation speed (dashed line in Fig. 4).

Despite experimental error, the off-rate measured directly and the one predicted by the Kramers' expression is in unexpectedly good agreement (Fig. 4), showing that Kramers' high-barrier approximation surprisingly holds under very high force, even under a force $f=0.447 \mathrm{pN}$, i.e., $f / f_{c}$ $\approx 0.91$, and an energy barrier of $\approx 1.9 k_{B} T$. We also calculated the off-rate given by mean first passage time (MFPT) approach for an absorbing boundary at the barrier location, and a reflecting boundary at the energy minimum. ${ }^{2}$ We obtain a predicted off-rate in good agreement with both experimental data and off-rate given by Kramers' theory (crosses in Fig. 4) by using a $33.5 k_{B} T$ zero-force energy barrier, which is the 
value in our landscape within experimental error on the zeroforce barrier height. Our point is not to perform a comparison between Kramers and MFPT approaches, since a more closer look would have then to be taken on boundary conditions used in the MFPT approach (which may not be perfectly reflecting nor absorbing). However, we conclude that given the experimental accuracy, both Kramers and MFPT approaches are in relatively good agreement and consistent with experimental data.

Distances involved in biomolecule bonds are orders of magnitude $\left(x_{b} \approx 0.1-1 \mathrm{~nm}\right)$ shorter than in the case of the minimalistic system studied here. The result of our study of a particular system may not be directly applicable to the study of biomolecule bonds. The condition of this applicability resides is the validity of the assumption that a single biomolecule bond can be described by a smooth energy profile. Limited experimental accuracy generally forbids a direct measurement of an energy profile at the molecular level, hence it is difficult to rule out the possibility that this condition is not fulfilled for some systems. However, smooth (1D) energy profiles have described successfully experimental data in several studies. ${ }^{6,7,15}$ We conclude that for the systems for which the above-mentioned assumption is valid, our present result justifies the use of Kramers' high-barrier approximation even close to the critical force, as done in recent studies. ${ }^{7,8}$ Furthermore, the high-force regime is likely to play a role when considering single biomolecule bond experiments. Indeed, typical values of $E_{b} \approx 5-30 k_{B} T$ and $x_{b}$ $\approx 0.1-1 \mathrm{~nm}$ (Refs. 6 and 15) lead to $f_{c} \approx 40-1200 \mathrm{pN}$, which are forces within the reach of AFM, BFP, or OTs in the lower part of this range. Dudko et al. ${ }^{7}$ obtained parameters for biomolecules studied in Refs. 16 and 17 showing that measured forces were close to the critical force in these cases. Finally, the novel approach presented here can be used in the future to either experimentally test theoretical predictions for thermally activated processes or address questions regarding colloidal behaviors.
In summary, we have shown that Kramers' approximation for high viscosity and high barrier holds even for a barrier height close to the thermal energy. We believe that this result is useful for strengthening the models used in the analysis of data obtained by measurements performed at high forces or low energy barrier.

We thank the Soft Condensed Matter group at Utrecht University for supplying the beads, C. Retif for technical support and K. Visscher, D. Frenkel, P. R. ten Wolde, and A. van der Horst for critical reading of the manuscript. We also thank P. D. J. van Oostrum, A. van der Horst, K. Visscher, S. Tanase-Nicola, T. Erdmann, and K. Sundyak for useful discussions. This work is part of the research program of the "Stichting voor Fundamenteel Onderzoek der Materie (FOM)," which is financially supported by the "Nederlandse Organisatie voor Wetenschappelijk Onderzoek (NWO)."

${ }^{1}$ H. A. Kramers, Physica (Amsterdam) 7, 284 (1940).

${ }^{2}$ P. Hänggi, P. Talkner, and M. Borkovec, Rev. Mod. Phys. 62, 251 (1990).

${ }^{3}$ R. Merkel, P. Nassoy, A. Leung, K. Ritchie, and E. Evans, Nature (London) 397, 50 (1999).

${ }^{4}$ G. I. Bell, Science 200, 618 (1978).

${ }^{5}$ E. Evans and K. Ritchie, Biophys. J. 72, 1541 (1997).

${ }^{6}$ S. Chen and T. A. Springer, Proc. Natl. Acad. Sci. U.S.A. 98, 950 (2001).

${ }^{7}$ O. K. Dudko, G. Hummer, and A. Szabo, Phys. Rev. Lett. 96, 108101 (2006).

${ }^{8}$ H.-J. Lin, H.-Y. Chen, Y.-J. Sheng, and H.-K. Tsao, Phys. Rev. Lett. 98, 088304 (2007).

${ }^{9}$ J. Husson and F. Pincet, Phys. Rev. E 77, 026108 (2008).

${ }^{10}$ A. Simon and A. Libchaber, Phys. Rev. Lett. 68, 3375 (1992).

${ }^{11}$ L. I. McCann, M. Dykman, and B. Golding, Nature (London) 402, 785 (1999).

${ }^{12}$ H. T. Schek and A. J. Hunt, Biomed. Microdevices 7, 41 (2005).

${ }^{13}$ L. P. Faucheux, G. Stolovitzky, and A. Libchaber, Phys. Rev. E 51, 5239 (1995).

${ }^{14}$ K. Visscher, S. P. Gross, and S. M. Block, IEEE J. Sel. Top. Quantum Electron. 2, 1066 (1996).

${ }^{15}$ F. Pincet and J. Husson, Biophys. J. 89, 4374 (2005).

${ }^{16}$ M. Carrion-Vazquez, A. F. Oberhauser, S. B. Fowler, P. E. Marszalek, S. E. Broedel, J. Clarke, and J. M. Fernandez, Proc. Natl. Acad. Sci. U.S.A. 96, 3694 (1999).

${ }^{17}$ J. Liphardt, S. Dumont, S. B. Smith, I. Tinoco, Jr., and C. Bustamante, Science 296, 1832 (2002). 